\title{
EL CURRÍCULO Y LA EDUCACIÓN A DISTANCIA
}

\author{
(THE CURRICULUM AND DISTANCE EDUCATION)
}

\author{
Walter Ruben Iriondo Otero \\ Universidad Federal de Pelotas (Brasil) \\ Domingo José Gallego Gil \\ Universidad Nacional de Educación a Distancia (España)
}

\section{RESUMEN}

Los procesos de enseñanza y de aprendizaje recibieron el impacto de las Tecnologías de la Información y Comunicación (TIC), que a la vez potenciaron a la Educación a Distancia (EaD) como modalidad importante de enseñanza y aprendizaje. Las TIC, especialmente los medios digitales soportados por la informática y por las redes de ordenadores, presentan la necesidad de re-posicionar los medios en el contexto del currículo y su integración curricular en EaD. En este trabajo escogimos un marco epistémico, y con esa lente académica identificamos los aspectos que debemos tener en cuenta al elaborar currículos para EaD. Ese marco de comprensión consideró al currículo como contenido a ser desarrollado, como la planificación de las actividades educativas, y como la realidad interactiva que subyace a la experiencia educativa. Para cada una de esas tres dimensiones identificamos aspectos del currículo que, a nuestro entender, deben ser considerados en EaD.

Palabras clave: currículo, educación a distancia, TIC, medios, tecnologías educativas.

\begin{abstract}
Teaching and learning processes have been strongly impacted by Information and Communication Technologies (ICTs). These have, in turn, maximized the potential of Distance Education (DE), i.e. as an important means of teaching and learning. ICTs, especially digital media that is supported by Information Technology and computer networks, require a (new) repositioning where both the curriculum context and the curriculum development in DE are taken into consideration. In this paper, we discuss an epistemological framework, which, when seen through this new academic lens, enables us to identify key aspects for developing curricula in DE. This framework considers the "curriculum" as content that is to be developed, namely the planning of educational activities and the interactive reality that underlies the
\end{abstract}


distance education experience. For each of these three dimensions, we identified key aspects of the curriculum which, in our view, should be carefully considered in DE.

Keywords: curriculum, distance education, ICT, media, educational technologies.

En la actualidad nos encontramos ante una nueva generación de estudiantes, "los nativos digitales", que han pasado toda su vida utilizando ordenadores, videojuegos, reproductores de música digital, cámaras de vídeo, teléfonos celulares y otras diversas herramientas de la era digital (Prensky, 2001). Ellos no tuvieron que acceder a las nuevas tecnologías, sino que nacieron con ellas y se posicionan ante el conocimiento desde postulados diferentes a los del pasado. Por ese motivo, y para incrementar la motivación de los alumnos y disminuir la evasión escolar, el diseño de los nuevos currículos y la práctica de la enseñanza deben considerar el análisis de las nuevas culturas juveniles (Marchesi, 2009).

Efectivamente, hasta hace algunos años, los procesos de enseñanza aprendizaje se organizaban alrededor del libro, de la cultura escrita, y del profesor como la gran fuente de transmisión del saber. Sin embargo, las Tecnologías de la Información y de la Comunicación (TIC) invadieron nuestro vivir cotidiano y lo modificaron profundamente. Al multiplicar los caminos para la obtención de la información, las TIC relativizaron el espacio escolar, como el local, privilegiado para la transmisión del saber; descolocaron al profesor de su posición de fuente primaria y central del conocimiento; y re-dimensionaron la cultura escrita al proveerle de nuevos materiales, no solo del libro. Las TIC proveen nuevos modelos de representar y decir el mundo mucho más allá del lenguaje oral, escrito, sonoro y de las imágenes fijas o en movimiento. Por lo tanto, podemos afirmar que las TIC presentan el desafío y la necesidad de repensar las prácticas pedagógicas tradicionales en su totalidad (Jacinski y Farako, 2002).

Observamos que el debate sobre TIC y currículo se organiza alrededor de los enfoques de "aprender sobre tecnologías" y "aprender con tecnologías". El enfoque de aprender sobre tecnologías se centra en el desarrollo de habilidades de manejo técnico de herramientas informáticas. El debate sobre TIC y currículo, cuyo enfoque es el de aprender con tecnologías, incorpora las competencias de selección y organización de informaciones, de comunicación, de trabajo en red o colaboración, así como las operaciones mentales, habilidades y actitudes implícitas en esas tareas (Padilha, 2009). 
En los últimos años las TIC, en particular las tecnologías digitales, catapultaron a la Educación a Distancia (EaD) como modalidad de enseñanza y de aprendizaje en Brasil. Ejemplos de la expansión de la EaD son producto del notable esfuerzo realizado por el Gobierno Federal, con la creación de la Universidad Abierta del Brasil (UAB) en el año 2005, y de la Escuela Técnica Abierta de Brasil (e-TEC) en el año 2007. Juntas, UAB y e-TEC reúnen en la actualidad centenares de millares de estudiantes que frecuentan cursos técnicos, de pre-grado y de postgrado en la modalidad EaD.

Si por un lado la expansión y la interiorización de la educación facilitada por la modalidad EaD van posibilitando democratizar el acceso a la educación técnica y superior, por otro lado, esa expansión exige un aumento de recursos y esfuerzos en investigación que tengan por objetivo la educación de calidad para los estudiantes matriculados en cursos ofrecidos en esa modalidad de enseñanza. En ese sentido, un asunto que despierta nuestro interés es el cuidado que debemos tener al elaborar los currículos cuando se trata de Educación a Distancia utilizando TIC, especialmente los medios digitales soportados por la informática y por las redes de ordenadores.

De hecho, la necesidad de re-posicionar los medios en el contexto del currículo y su integración curricular se convierte en una cuestión importante y primordial no solo en el aspecto analítico, descriptivo o detallado de los medios, sino también por las posibilidades que ellos ofrecen a la hora de funcionar en determinado programa pedagógico. Es desde el campo curricular y desde las diferentes concepciones curriculares donde mejor podemos comprender las distintas acepciones a la hora de seleccionar recursos tecnológicos (Ballesta Pagán, 2011).

Pero cuando intentamos profundizar el estudio en ese campo, observamos que el concepto de currículo es probablemente uno de los más controvertidos dentro de los que normalmente se encuentran en cualquier análisis disciplinar del área de la educación. Llegar a un consenso al conceptuar currículo es un arduo desafío, vista la diversidad de autores que abordan el tema según criterios variados (Angulo, 1994; Sacristán y Gómez, 1998). Ballesta Pagán (2011) opina que no existe una única forma de considerar el currículo y en particular, en lo que respecta a las TIC, cada concepción curricular plantea una visión muy particular y diferente sobre los medios, y sobre los diferentes modos de considerarlos, tanto en la teoría como en la práctica.

Resulta claro que la comprensión de lo que entendemos por currículo depende de marcos variados (Angulo, 1994; Gimeno Sacristán y Pérez Gómez, 1998; Moreira y Candau, 2007; Ballesta Pagán, 2011) y que, por lo tanto, su estudio bajo la óptica de los medios digitales en la educación puede llevar en consideración diferentes 
lentes epistémicas a través de las cuales abordamos los problemas que conciernen al tema. En este capítulo escogeremos específicamente un marco de comprensión para ese asunto, y con esa lente académica intentaremos identificar los cuidados que debemos tener al elaborar currículos para la Educación a Distancia.

\section{EL CURRÍCULO EN LA EDUCACIÓN A DISTANCIA}

Entre las muchas definiciones existentes, vamos a considerar la que define Educación a Distancia como la modalidad educacional en la cual la mediación didáctico-pedagógica en los procesos de enseñanza y de aprendizaje ocurren con la utilización de medios y tecnologías de información y comunicación, con estudiantes y profesores desarrollando actividades educativas en lugares o tiempos diversos (Decreto presidencial 5.622, de 19 de diciembre de 2005, SEED/MEC/Brasil).

Pero no podemos olvidarnos de que el concepto de Educación a Distancia no es algo nuevo, ya que podríamos decir que existe desde el inicio del libro impreso, y fue sufriendo transformaciones en la medida en que las innovaciones tecnológicas se fueron incorporando a la sociedad. En ese sentido, Anaraki (2004) y Jisc (2004) comentan que a partir de los años 9o, con el sensible avance del uso de las TIC en el proceso de enseñanza y de aprendizaje, fueron fortalecidas en la EaD las dimensiones de la conectividad, flexibilidad, interacción, autonomía, colaboración, motivación, economía, además de crearse oportunidades para nuevos abordajes para el proceso de enseñanza y de aprendizaje (Cuadro 1).

\begin{tabular}{|l|l|}
\hline Dimensiones & \multicolumn{1}{|c|}{ Descripción } \\
\hline Conectividad & $\begin{array}{l}\text { El acceso a la información está disponible en escala global. El conocimiento } \\
\text { almacenado puede ser actualizado a cualquier momento. }\end{array}$ \\
\hline Flexibilidad & El aprendizaje puede acontecer a cualquier hora y en cualquier lugar. \\
\hline Interacción & Evaluaciones pueden ser realizadas de forma autónoma e inmediata. \\
\hline Autonomía & Promueve autonomía en el aprendizaje. \\
\hline Colaboración & $\begin{array}{l}\text { El aprendizaje colaborativo es facilitado por aplicaciones como foros, } \\
\text { mensajes instantáneos, conferencias. }\end{array}$ \\
\hline Motivación & El uso de recursos multimedia puede tornar el aprendizaje más divertido. \\
\hline Economía & Economía para las instituciones (a largo plazo). \\
\hline $\begin{array}{l}\text { Nuevas } \\
\text { oportunidades }\end{array}$ & Ofrece nuevos abordajes para la enseñanza y el aprendizaje. \\
\hline
\end{tabular}

Cuadro 1. Beneficios de la Educación a Distancia utilizando TIC

Fuente: JISC (2004) y Anaraki (2004) 
Breitner y Hoppe (2005) comentan que los cambios de las condiciones tecnológicas, y el desarrollo y perfeccionamiento de nuevas tecnologías, llevan consigo la necesidad de desarrollar modelos de referencia para la EaD, con foco en las dimensiones pedagógica y tecnológica.

Efectivamente, no podemos permanecer insensibles ante las posibilidades ofrecidas por las TIC para tornar viable el nuevo paradigma del proceso de enseñanza y de aprendizaje. Downes (2004) y Fox (2005) afirman que en ese nuevo paradigma, el proceso de aprendizaje basado en el estudio de contenidos cede espacio para el abordaje del aprendizaje basado en experiencias. De esa forma, el aprendizaje es concebido como una fuente continua, sobre demanda, disponible en el lugar y en el momento que sea necesaria, y se concreta por la interacción y aplicación, no solo por la distribución de informaciones. Las redes de ordenadores, notablemente la red de Internet, posibilitan navegar a través de una red de conocimientos, en lugar de moverse apenas a lo largo de una cadena de informaciones. Hay, también, una expectativa creciente de que el aprendizaje se configure para las preferencias personales, en lugar de tener soluciones generales para todas las personas, privilegiando de esa forma la diversidad en muchos aspectos. Considerando las opciones de combinación de aprendizaje ofrecidas por las TIC, la responsabilidad de la calidad de la experiencia de aprendizaje no le compete solo al profesor, sino también al estudiante. En ese contexto, el estudiante pasa de simple receptor para asumir más responsabilidad en el proceso, contribuyendo a la construcción del conocimiento.

Por lo tanto, es razonable suponer que el nuevo paradigma del proceso de enseñanza y de aprendizaje exija repensar el currículo para la Educación a Distancia. Ballesta Pagán (2011) alerta que el discurso que desarrolla la concepción curricular crítica en torno de los medios destaca algunos aspectos que no deben ser ignorados. Por un lado, los medios actúan como elementos facilitadores del análisis, pensamiento crítico y transformación de las prácticas, contenidos e informaciones que representan los intereses políticos, ideológicos y económicos que condicionan el mensaje. Por lo tanto, resulta evidente la necesidad de revisar la función transformadora y reproductora de los medios mediante la reflexión en torno al tipo de sociedad y a los valores éticos que promueven los auténticos beneficios del mensaje. Por otro lado, es necesario contemplar la función emancipadora de los medios, practicar la resistencia y utilizarlos en la tarea de detectar y plantear problemas, dotándolos de la posibilidad de la transformación social. Para alcanzar ese objetivo es necesario una profunda conciencia y compromiso, así como dominar los medios tanto en el aspecto técnico, como en el ideológico. 
Ballesta Pagán (2011) continúa su explicación diciendo que es posible distinguir en el currículo una orientación técnica, práctica y crítica que lleva asociada el uso de los medios que puede ser transmisor/reproductor, práctico/situacional, y/o crítico/transformador. El uso transmisor/reproductor de los medios está orientado por la racionalidad tecnológica (perspectiva positivista). En esta perspectiva, los medios están al servicio del control técnico de procesos objetivos y mensurables. El uso práctico/situacional de los medios está orientado sobre la estructura de la racionalidad hermenéutica y fenomenológica. Los medios son los canales que, al interpretar y explicar la realidad, posibilitan reconstruir significativamente los procesos de enseñanza y de aprendizaje. El uso crítico/transformador de los medios parte de la estructura de la racionalidad crítica o emancipadora para dar un paso más hacia la busca del entendimiento interpretativo. Conviene entender que el currículo es social, cultural y políticamente determinado, y defender la reflexión y deliberación crítica de la práctica para mejorarla, transformando a su vez los códigos y principios que orientan la selección de contenidos y su organización, disminuyendo las desigualdades sociales y las dificultades en la enseñanza (Ballesta Pagán, 2011).

Dicho esto, y para sumergirnos en el asunto que nos interesa, el currículo bajo la óptica de la Educación a Distancia, debemos elegir una definición de currículo que oriente la discusión. Realizar esa elección resulta difícil ante la diversidad de enfoques del tema disponibles en la literatura. Gimeno Sacristán y Pérez Gómez (1998) apuntan la dificultad de ofrecer una definición válida de currículo que sea aceptada universalmente. La comprensión de currículo depende de marcos variables para concretizar su significado.

Con la finalidad de ejemplificar la diversidad de asuntos abordados en el estudio del currículo, vamos a citar inicialmente la definición de Gimeno Sacristán y Pérez Gómez (1998) que conceptúan currículo como el eslabón entre la cultura, la sociedad exterior a la escuela y la educación, entre el conocimiento o la cultura heredada y el aprendizaje de los alumnos, entre el mundo y la teoría, de las ideas, de los supuestos, aspiraciones, y la práctica posible, dadas unas condiciones determinadas. Es posible observar la amplitud de la definición y queda clara la gran responsabilidad de quien pretenda trabajar en esa área.

Moreira y Candau (2007) comentan que a la palabra currículo se asocian distintas concepciones, que derivan de los diversos modos de cómo la educación es concebida históricamente, así como de las influencias teóricas que la afectan y se hacen hegemónicas en un momento dado. Factores socio-económicos, políticos y culturales contribuyen para que el currículo sea entendido como: a) los contenidos 
que deben ser enseñados y aprendidos; b) las experiencias de aprendizaje escolares que deben ser vividas por los alumnos; c) los planos pedagógicos elaborados por profesores, escuelas y sistemas educativos; d) los objetivos que deben ser alcanzados por medio del proceso de enseñanza; e) los procesos de evaluación.

Angulo (1994) opina que los diferentes conceptos de currículo pueden ser agrupados en tres apartados fundamentales: 1) currículo como contenido;2) currículo como planificación; 3) currículo como realidad interactiva. En el primer apartado, Angulo (1994) agrupa autores que entienden el currículo como el conocimiento disciplinar que debe ser estudiado, la experiencia educativa que aporta la escuela, el desarrollo de procesos cognitivos, el material de aprendizaje, una serie estructurada de resultados de aprendizaje que prescribe, o al menos anticipa, los resultados de la educación. El conocimiento que el alumno aprende no está enteramente comprendido por el contenido seleccionado, sino también por todos los significados, patrones de conducta, valores morales, etc. que subyacen la experiencia educativa, o que, por el contrario, en ella son prohibidos u obviados.

En el segundo apartado, Angulo (1994) agrupa los autores para los cuales el concepto de currículo significa planificación, inevitablemente asumiendo que en él viene establecido explícitamente el marco dentro del cual se desarrollarán las actividades educativas de una institución de enseñanza. Un conjunto organizado de intenciones educativas y de entendimiento, donde se presenta tanto lo que ha de ser aprendido y enseñado, como los materiales, los métodos de enseñanza, etc. El currículo debe mostrar la relación entre elementos tales como enunciado de las intenciones, objetivos, contenido curricular para alcanzar los objetivos, enunciados sobre los esquemas de evaluación del currículo. El currículo como planificación, además de retomar las concepciones anteriores, dado que inevitablemente ha de incluir el contenido educativo, señala el marco ideal dentro del cual se desenvolverá la actividad educativa. El currículo operativo, o en uso, es el resultado de llevar a la práctica las prescripciones del currículo planificado.

En el tercer apartado, Angulo (1994) congrega a los autores que entienden que si el currículo es determinado, y a la vez determina la vida escolar, el conocimiento de cómo es vivido, creado y traducido de forma interactiva en las clases, por docentes y alumnos, resulta esencial para mejorar sus prescripciones. Si el currículo es también, y fundamentalmente, lo que acontece en los salones de clase, se hace necesaria una nueva perspectiva que centre su interés en las conexiones, o des-conexiones, entre el currículo como intención, y el currículo como acción a través del papel creativo del docente. 
La discusión que proponemos en este capítulo sobre el currículo y la Educación a Distancia estará pautada por los apartados enunciados por Angulo (1994). O sea, abordaremos aspectos que entendemos deben ser considerados en el currículo en lo que respecta al contenido, a la planificación y a la realidad interactiva en la Educación a Distancia.

\section{EL CONTENIDO}

A pesar de ser una visión limitada, el currículo es visto popularmente como el contenido o conocimiento valioso y digno de ser aprendido. Otros autores asocian el significado del currículo con el conocimiento disciplinar a ser estudiado, con la experiencia educativa que aporta la institución educativa, con el desarrollo de procesos cognitivos, con el material de aprendizaje, y con una serie estructurada de resultados de aprendizaje que prescribe (o al menos anticipa) los resultados de la instrucción (Taylor y Richards, 1979 en Angulo, 1994).

Moreira y Candau (2007) afirman que el currículo puede ser entendido como las experiencias de aprendizaje escolares que deben ser vividas por los alumnos. Recordemos aquí los cursos administrados en la modalidad de Educación a Distancia soportados por medios digitales, las experiencias de aprendizaje ofrecidas a los alumnos en los Ambientes Virtuales de Aprendizaje (AVA).

Los AVA son sistemas informáticos que combinan diversos medios para ofrecer a los alumnos tanto disciplinas aisladas como cursos completos, integrando con fines educativos diversas herramientas de comunicación ya existentes en las redes de ordenadores, especialmente la red de Internet. En los AVA, el profesor puede colocar a disposición de los alumnos, de forma estructurada e integrada, textos, hipertextos, imágenes, sonido, vídeo y animaciones en dos o tres dimensiones.

Pero el hecho de trabajar con medios digitales no significa olvidarnos que el lenguaje y la educación son inseparables. Gimeno Sacristán (2002) afirma que la escolaridad debe ocuparse ante todo, del hablar y del escuchar, del leer y del escribir. Cultivar esas dos últimas habilidades es función esencial de la educación moderna, pues son elementos para penetrar a la cultura y por ella ser penetrado. La lectura alcanza un valor decisivo en la construcción del espacio de la subjetividad, de la individualidad, del pensamiento abstracto, crítico y reflexivo, y de la autonomía del hombre y de la mujer modernos. Las TIC no substituyen estas prácticas culturales, sino que parten de ellas y las necesitan. Solamente los buenos lectores pueden extraer de las TIC sus mejores posibilidades (Gimeno Sacristán, 2002). En ese 
sentido, una experiencia clásica de aprendizaje en los AVA consiste en solicitar que los estudiantes realicen lecturas y posteriormente participen en foros para debatir un asunto de interés de la asignatura, donde los estudiantes deben posicionarse y respaldar sus ideas en los textos leídos previamente.

Reforzando esa idea, Garrison y Anderson (2003) consideran que la gran ventaja de la EaD consiste en su capacidad de soportar interacciones asíncronas basadas en texto, que posibilitan la reflexión, independiente de la presión del tiempo y de las restricciones impuestas por la distancia. La interacción acontece entre el estudiante y el profesor, entre el estudiante y el material de estudio, y entre el estudiante y sus pares. El uso de los medios disponibles en los AVA para implementar las referidas interacciones ofrece ventajas que no estaban presentes en modalidades anteriores de Educación a Distancia. Además, acompañando los registros en foros, envío de mensajes instantáneos y correo electrónico, las interacciones de cada estudiante pueden ser analizadas y medidas, impactando en los resultados del aprendizaje.

Si por un lado nuestra experiencia docente nos muestra que los debates en las clases presenciales son, con frecuencia, polarizados por unos pocos alumnos del grupo, muchas veces inhibiendo otros alumnos de participar de la discusión; por otro lado, en los AVA, el debate de cierto asunto puede ser definido como un foro de participación obligatoria, exigiendo del estudiante la reflexión, el pensamiento crítico y su posicionamiento frente al profesor y sus colegas. Por lo tanto, el uso de Ambientes Virtuales de Aprendizaje ofrece experiencias de aprendizaje que no siempre son observadas en las clases presenciales.

Sucede, sin embargo, que el simple hecho de que los AVA ofrezcan diversos recursos educativos no significa que los mismos sean utilizados, o que lo sean de forma apropiada. Los profesores pueden utilizar el AVA apenas como un repositorio de informaciones, textos, diapositivas, imágenes y vídeos, sin que eso agregue valor a las experiencias de aprendizaje de los alumnos. Por el contrario, esa actitud puede perjudicar la imagen de esa y de otras iniciativas de Educación a Distancia. Pero entonces, ¿qué más debe ser considerado en el currículo para obtener experiencias de aprendizaje exitosas en EaD?

Nuestra praxis profesional nos lleva a creer que algunos de los aspectos que deben ser considerados para garantizar las experiencias exitosas en EaD pasan por la elección adecuada de los materiales de estudio colocados a disposición de los estudiantes, por las tareas propuestas, por los ejercicios propuestos como actividades prácticas, y por las oportunidades de evaluación formativa y sumativa. 
Las TIC posibilitan al profesor el desarrollo de materiales de estudio más sofisticados y, por lo tanto, puede proponer a los estudiantes actividades de enseñanza y de aprendizaje también más sofisticados. En la medida que los AVA están incorporando los recursos disponibles en Internet, los profesores tienen en sus manos la posibilidad de "cautivar" a los estudiantes, proponiendo actividades que despierten su curiosidad, motivándolos al estudio autónomo.

Como sucede en la educación presencial, cuando se diseña una tarea para la Educación a Distancia, es necesario tener presente los objetivos que deben ser alcanzados por los estudiantes. Existen diversas taxonomías para auxiliar al profesor en la clasificación de los objetivos educacionales y para expresarlos de forma clara. Una de esas taxonomías más clásicas, frecuentemente citada en artículos científicos, es la Taxonomía de Objetivos Educacionales propuesta por Bloom en el año 1956, que divide los objetivos educacionales en tres dominios: afectivo, psicomotor y cognitivo. Este último dominio incluye los objetivos educacionales que tratan del conocimiento $\mathrm{y}$ del desarrollo de las habilidades intelectuales.

También defendemos la idea de que las actividades de enseñanza y de aprendizaje propuestas en EaD deben aprovechar las posibilidades de interacción ofrecidas por las TIC. Cuando hablamos de interacción no nos referimos solo a la interacción del estudiante con sus colegas o con el profesor, sino que también nos referimos a la interacción del estudiante con los materiales de estudio y a la interacción del estudiante consigo mismo. En la Teoría de la Conversación, Pask (1976), afirma que el aprendizaje acontece a través de interacciones recursivas (que él llama de conversaciones), respecto del asunto estudiado. Esas conversaciones sirven para que el conocimiento se torne explícito. Durante las conversaciones, las diferencias entre los participantes se reducen, hasta llegar a un acuerdo entre las partes, que Pask denomina de "acuerdo sobre el entendimiento". Laurillard (2002) afirma que el proceso de enseñanza y de aprendizaje debe acontecer como un diálogo interactivo, discursivo, adaptativo y reflexivo, y debe operar en el nivel de la descripción de los tópicos y en el nivel de las acciones relativas entre las tareas. Cuando trabajan en un ambiente on-line, los estudiantes, aunque estén distantes geográficamente, tienen la posibilidad de realizar tareas de forma colaborativa, compartiendo informaciones y ofreciendo soporte, tanto en forma de opiniones como de evaluaciones hechas por los propios colegas. Coincidimos con Pask (1976) y Laurillard (2002), entendiendo que las actividades propuestas en los AVA deben considerar experiencias de aprendizaje que contemplen la interacción y la construcción colectiva del conocimiento. 
Las tareas que envuelven conversaciones asíncronas, por ejemplo al debatir un texto en un foro, posibilitan que los estudiantes piensen sobre el asunto debatido, analicen las ideas de sus colegas y profesores, reconsiderando lo que están escribiendo, revisando los términos utilizados, teniendo más cuidado al componer sus ideas. Esto exige que los estudiantes estén más atentos respecto del tema en debate, más reflexivos y críticos en relación a su propio trabajo y al trabajo de los otros. El asincronismo en la comunicación, o sea, la demora entre el envío de un mensaje y la obtención de la respuesta, ofrece la posibilidad de reflexionar sobre el asunto debatido, motivando, de esa forma, el desarrollo de la habilidad de la reflexión en los estudiantes.

Otra característica de los Ambientes Virtuales de Aprendizaje es que ofrecen a los profesores la posibilidad de utilizar diversos medios para presentar el material instructivo y dar soporte al aprendizaje. Utilizando TIC es posible ofrecer en el AVA textos, figuras, diseños, vídeo y audio conferencias, música, animaciones, etc. Las TIC le posibilitan al estudiante interactuar con los productos pedagógicos utilizados en la educación, manipulando objetos virtuales que facilitan el aprendizaje de nuevos conceptos.

Una de las características de la EaD utilizando TIC, especialmente las tecnologías digitales, consiste en la incorporación de materiales didácticos poco frecuentes en las clases presenciales. Por ejemplo, materiales que contemplen la manipulación de imágenes en tres dimensiones, simulaciones, definición de múltiples escenarios, y muchos otros recursos que pueden ser utilizados con fines pedagógicos. Como veremos de forma más detallada en el apartado de planificación, utilizando TIC es posible desarrollar ejercicios de auto-evaluación, para que los estudiantes puedan verificar su evolución en la construcción del conocimiento. Existen softwares de autor que pueden ser utilizados por los profesores para realizar ejercicios de evaluación formativa, de tipo falso o verdadero, respuestas múltiples, llenar los espacios, palabras cruzadas, etc. Esos ejercicios pueden ser incorporados al AVA para que los alumnos evalúen de forma autónoma la evolución de su aprendizaje. Las actividades de evaluación formativa son importantes para indicar los aspectos teóricos que deben ser reforzados, estimulando de esa forma el interés y la motivación de los alumnos.

Estas facilidades ofrecidas por las TIC posibilitan el desarrollo de materiales y actividades educacionales sofisticadas para la EaD; actividades éstas que serían prácticamente imposibles de ser desarrolladas por el profesor en una clase presencial convencional, utilizando solo la tiza y el pizarrón. 


\section{LA PLANIFICACIÓN}

A pesar de que asociar el currículo al contenido a ser enseñado sea el significado más popularizado del currículo, Tyler (1973), citado por Angulo (1994), introduce un matiz importante, al afirmar que currículo es el trasfondo que subyace tanto en las actividades de planificación, como en los procesos de enseñanza y de aprendizaje. El currículo engloba los propósitos que guían la acción, así como la acción propiamente dicha. Esto quiere decir que el análisis adecuado del currículo debe extenderse desde un extremo propiamente prescriptivo hasta otro propiamente interactivo. Cuando el concepto de currículo significa planeamiento, inevitablemente se asume que en él viene establecido de forma explícita el marco dentro del cual se desarrollarán las actividades educativas de una escuela (Angulo, 1994).

La EaD utilizando TIC ofrece diversas ventajas, como indicamos antes, pero también presenta dificultades que deben ser contempladas. En los cursos presenciales, en cuanto los estudiantes se sientan en sus asientos, el profesor puede comenzar la clase de inmediato. Pero, cuando se trata de $\mathrm{EaD}$, para entrar en el salón de clase, los alumnos necesitan utilizar recursos técnicos, navegar en el ambiente virtual de aprendizaje, localizar el material de estudio necesario para realizar las actividades, etc. Esto exige de los estudiantes habilidades para el uso de recursos informáticos. Necesitan saber cómo utilizar el ordenador y sus periféricos, dialogar con el sistema operativo, utilizar diversos programas, navegar en ambientes virtuales. A pesar de que los alumnos de las nuevas generaciones son nativos digitales, no podemos suponer que todos ellos llegan al curso habiendo desarrollado previamente las habilidades necesarias para estudiar en un AVA. Por lo tanto, la capacitación en el uso de esas herramientas debe ser contemplada en la planificación de los cursos en la modalidad de EaD.

Otro aspecto que no puede ser ignorado en la planificación de la EaD es el hecho de que estudiar a distancia exige un esfuerzo adicional, tanto para quien enseña como para quien aprende. El profesor necesita estar capacitado para desarrollar materiales y estrategias de enseñanza adecuadas a la EaD. El estudiante, por su parte, precisa desarrollar habilidades cognitivas específicas, recibir soporte adecuado, y acceder a materiales didácticos que ofrezcan las experiencias de aprendizaje deseadas. La Educación a Distancia exige un cambio en el foco del proceso de enseñanza y de aprendizaje, en el papel representado por el profesor y en las responsabilidades designadas a los alumnos, de quien se espera autonomía en el estudio y participación activa en el proceso y en la construcción del conocimiento. 
Sucede que, como afirma Long (1990), no es razonable esperar que personas que durante todos los años de su vida escolar estuvieron acostumbradas a recibir la información elaborada y formateada, se transformen instantáneamente en estudiantes autónomos. Al elaborar currículos para la EaD, debemos tener presente que para ser autónomo, el estudiante debe aceptar, de forma consciente, la responsabilidad de tomar decisiones respecto de las metas y del esfuerzo a ser realizado, transformándose, por lo tanto, en su propio agente del cambio de paradigmas.

El estudio autónomo se torna crítico, de acuerdo con Fischer y Scharff (1998), cuando pasa a ser una parte integrante de la vida de las personas, derivada del deseo o de la necesidad de entender alguna cosa, o para poder realizar alguna actividad. Boud, Keogh y Walker (1985) sugieren que la habilidad del estudio autónomo puede ser adquirida, y proponen una escala o línea de progresión que va de la dependencia a la independencia, y después a la interdependencia. Cada una de esas etapas requiere que los estudiantes sean capaces de reflexionar y evaluar sus propias habilidades y capacidades.

Por lo tanto, la planificación del aprendizaje en EaD debe contemplar, también, el desarrollo en los alumnos de la habilidad del estudio autónomo. El currículo puede facilitar el desarrollo de la habilidad del estudio autónomo de los estudiantes si deliberadamente les transfiriese la responsabilidad del aprendizaje. El currículo puede prever diferentes niveles de estudio autónomo, incluyendo, por ejemplo, libertad de elección en el momento de determinar límites y objetivos. Candy (1991) comenta que el desafío del desarrollo de la habilidad del estudio autónomo consiste en llegar a un punto de equilibro, ofreciendo la correcta cantidad de ayuda sin dar demasiadas orientaciones. Esta dirección en exceso atenta contra el sentimiento de pertenencia que los estudiantes tienen en relación al proceso de aprendizaje. Honey y Mumford (2000) sugieren que los profesores deben disminuir gradualmente la cantidad de ayuda y dirección en la medida que los estudiantes van adquiriendo madurez y sintiendo más confianza en el proceso de aprendizaje.

Otro aspecto que debe ser considerado en el currículo son las diversas formas de aprender de los alumnos. Como hemos mencionado antes, las posibilidades ofrecidas por las TIC, notablemente las tecnologías de comunicación digital, refuerzan la expectativa de que el proceso de enseñanza y de aprendizaje sea configurado para las preferencias personales de cada alumno, en lugar de tener soluciones generales para todos ellos (Downes, 2004; Fox, 2005). 
Efectivamente, las aplicaciones prácticas de la metodología de los Estilos de Aprendizaje para la Educación a Distancia son múltiples (Alonso, Gallego y Honey, 2007; García Cué, 2006), y hay autores, como es el caso de Melaré (2011), que culpan el fracaso de algunos adultos en la $\mathrm{EaD}$ a la falta de adecuación del estilo de aprendizaje del alumno y los materiales didácticos que debe estudiar.

En este sentido, contemplar el estudio de los estilos de aprendizaje de los alumnos en el currículo puede ser un elemento determinante en el proceso de adaptación y mejora de la Educación a Distancia. Por un lado, un buen diseño de instrucción debe tener en cuenta el análisis de necesidades de aprendizaje y, por consiguiente, conocer los estilos de aprendizaje predominantes de los alumnos, repercutirá significativamente en ese diseño (los contenidos, la metodología, el ritmo, podrán ser los adecuados de acuerdo al marco de aprendizaje que generan los estilos diagnosticados).

Por otro lado, también debemos pensar qué tipo de materiales didácticos y qué recursos tecnológicos elegimos como más adecuados para nuestros alumnos. Utilizar materiales impresos o recursos informáticos o audiovisuales favorecerá más a un tipo de alumnos con preferencias en algún estilo de aprendizaje determinado, y no ayudará a otros alumnos con preferencias acusadas por otros estilos de aprendizaje. Tratemos de averiguar, entonces, a qué estilos de aprendizaje de los alumnos favorecen los materiales impresos, y a quién los recursos tecnológicos, para poder seleccionar en cada situación los recursos más adecuados. Si los resultados del diagnóstico de un grupo determinado de alumnos son positivos, el profesor podrá utilizar estos medios tecnológicos con el convencimiento de que sus alumnos van a aprender mejor.

Si la aplicación de las TIC es de uso individualizado, como pueden ser las aplicaciones multimedia, el ajuste al estilo de aprendizaje del alumno es muy conveniente. No realizar este ajuste puede conducir a que el aprendizaje no alcance el ritmo y efectividad deseados. Se trataría, en definitiva, de ofertar distintas posibilidades al discente para que desarrolle, en lo posible, su capacidad de aprender a aprender.

Asimismo, la aplicación de la metodología de los estilos de aprendizaje nos llevaría a reconceptualizar la forma y metodología de las evaluaciones realizadas a los alumnos. Cada materia debería ser analizada y contrastada dentro de este marco de reflexión, lo que posibilitaría la elaboración de un tipo de evaluación más ajustada a los contenidos de aprendizaje. 
También, desde el punto de vista del alumno, este discurso es de máxima utilidad. La "soledad" del discente a distancia le pide un mayor componente de autoconocimiento para salir airoso de las dificultades de aprendizaje. Solo conociendo cómo aprenden nuestros alumnos podremos orientarles eficazmente en sus propios procesos de aprendizaje, enseñándoles a emplear aquellos estilos más adecuados en cada situación, ayudándoles a "aprender a aprender".

Por último, el conocimiento de los estilos de aprendizaje aumenta la eficacia de la acción tutorial. El tutor que haya analizado el estilo de aprendizaje predominante en su grupo tutorial contará con un recurso científico a la hora de enfocar técnicas de estudio de sus alumnos, adecuadas a cada materia y, si además conoce el estilo de aprendizaje predominante de cada alumno, realizará mejor su labor en la tutoría individual. Por ejemplo, si la mayoría de la clase tiene como estilo preferido el estilo reflexivo (facilidad para aprender y expresarse en todo lo que signifique analizar, almacenar datos, desmenuzar contenidos), el tutor sabrá qué tipo de estudio es el más fácil para los alumnos. Y, si no fuera así, sino que las materias exigen mucho trabajo de análisis y ese grupo está muy bajo en ese estilo, la labor del tutor será intentar mejorar el estilo reflexivo por medio de tareas y actividades que exijan ese estilo.

Los estilos de aprendizaje son relativamente estables. Esto quiere decir que cada uno de nosotros tiene una configuración propia de preferencias y estilos de aprendizaje, pero esa configuración va evolucionando a lo largo de la vida. De hecho el profesor puede y debe modelar y mejorar las preferencias de estilos de aprendizaje de sus alumnos. Tiene que ayudarles, primero dándoselos a conocer y después facilitándoles el dominio de los mismos, con el fin de que acaben siendo capaces de emplear un estilo u otro según lo requiera la situación. Para ello cuenta con una amplia serie de actividades y sugerencias a las que nos hemos referido con amplitud en otra ocasión (Alonso, Gallego y Honey, 2007).

El profesor puede ayudar a mejorar las preferencias de estilos de aprendizaje de sus alumnos utilizando diversos recursos y estilos de enseñanza (Martínez Geijo, 2007), empleando de diversas maneras los materiales didácticos, que exijan diversas formas de captar y procesar la información.

Por último, señalar que detrás de la utilidad de la metodología de los estilos de aprendizaje no se esconde la exigencia de acomodación, por parte del docente, a las preferencias de estilo de todos los alumnos en todas las ocasiones, sino un esfuerzo por su parte para comprender las diferencias en los estilos de aprendizaje de sus 
alumnos; cambiar su estilo de enseñar en aquellas áreas y en aquellas situaciones que lo requieran, cuando esto sea posible; y ayudar a los estudiantes a desarrollar todos sus posibles estilos de aprendizaje, facilitándoles su propio autodiagnóstico y favoreciendo y fomentando aquellos estilos en los que tengan preferencias más bajas.

Otro aspecto de planificación que debe ser previsto son las actividades de evaluación. Como ya dijimos antes, una de las facilidades ofrecida por la EaD apoyada en AVA, al compararla con la enseñanza presencial, consiste en la facilidad de elaborar y disponer de actividades de evaluación formativa on-line. Esa es una característica que debe ser contemplada en el currículo. Las evaluaciones formativas on-line, o sea, actividades propuestas a lo largo del proceso de aprendizaje que posibilitan la auto-evaluación por parte de los estudiantes, deben ser consideradas en el currículo no solo como instrumentos de evaluación, sino que también deben formar parte de la estrategia de construcción del conocimiento.

Robinson y Udall (2003) comentan que las estrategias de evaluación deben ser diseñadas de forma que estimulen en los estudiantes las habilidades de identificar, estructurar y articular preguntas relativas a su propio entendimiento del asunto objeto de estudio. Las auto-evaluaciones durante el aprendizaje pueden desempeñar un papel extremadamente importante en ese proceso, siempre que el foco esté dirigido a lo que "los estudiantes hacen", y no a lo que "los profesores piensan que ellos hacen". El desafío consiste en alcanzar ese objetivo con un esfuerzo razonable, tanto por parte de los estudiantes, cuanto por parte de los profesores.

Por otro lado, partiendo del presupuesto de que las interacciones de los estudiantes en los foros en el AVA reflejan el aprendizaje que van desarrollando a lo largo del curso, el currículo debe considerar la evaluación de la participación de los alumnos en los referidos foros. Rosa y Maltempi (2006) afirman que en las aplicaciones de mensajes instantáneos y en los foros, en los cuales hay un gran número de interacciones entre el estudiante con el profesor y con sus colegas, el participante se torna agente de la construcción de su propio conocimiento y la calidad de sus manifestaciones debe ser evaluada.

Algunos autores plantean modelos para evaluar la calidad de las manifestaciones de los estudiantes en los foros del AVA. Es el caso de Garrison y Anderson (2003), que sugieren que la calidad de las manifestaciones de los estudiantes en los foros de un AVA pueden ser evaluadas observando si las referidas manifestaciones estimulan a otros estudiantes a participar y aprender, si contribuyen con los puntos importantes 
del debate, si crean un ambiente amigable para el aprendizaje, si toman la iniciativa de responder a otros estudiantes, si procuran incluir otros estudiantes en el debate, si traspasan barreras personales para participar del debate si demuestran reflexión y si utilizan el AVA de forma original para incrementar el propio aprendizaje y el aprendizaje de los otros alumnos. Por su parte, Ho y Swan (2007) presentan un método para evaluar los mensajes incluidos en los foros, donde las manifestaciones de los estudiantes son analizadas en función de las dimensiones: calidad, relevancia, cantidad y forma. Estos y otros métodos son abordados con más profundidad en Iriondo Otero (2008), e Iriondo Otero y Ribeiro (2010).

El soporte a los estudiantes es otro aspecto que también debe ser considerado en el currículo de cursos ofrecidos en la modalidad de Educación a Distancia. Sin embargo, Simpson (2002) sostiene que aparentemente el soporte a los estudiantes en EaD no ha recibido la atención que merece. Tal vez eso se deba a que el soporte a los estudiantes sea una actividad de poco glamour en el universo de la Educación a Distancia, o tal vez porque los actuales profesores de los cursos de EaD tienen poca (o ninguna) experiencia participando como alumnos en cursos a distancia, y por lo tanto les resulta difícil comprender la importancia que el soporte tiene para los estudiantes en esa modalidad de aprendizaje (Simpson, 2002).

Hitch y MacBrayne (2003) comentan que los estudiantes de cursos en la modalidad EaD con frecuencia experimentan aislamiento, y no han recibido los mismos servicios académicos y de soporte que están disponibles para sus colegas matriculados en cursos presenciales. Ese aislamiento puede inhibir la comunicación y ocasionar la evasión de los estudiantes.

El currículo debe considerar el soporte a los estudiantes en sus diferentes dimensiones. Simpson (2002) clasifica el soporte en dos categorías. La primera es el soporte académico, y está relacionado con el soporte a los estudiantes en lo que respecta a las cuestiones cognitivas, intelectuales y del conocimiento. La segunda categoría se refiere al soporte no académico, que trata de aspectos organizativos y afectivos. También Hitch y MacBrayne (2003) afirman que el soporte on-line no debe considerar apenas aspectos relativos al contenido educativo, sino que debe contemplar toda la problemática relacionada con la vida estudiantil.

Sin embargo, ofrecer soporte efectivo a los estudiantes de cursos administrados en la modalidad de $\mathrm{EaD}$, significa conectar sectores de las instituciones de enseñanza que hasta ese momento no estaban íntimamente relacionados. Sectores como tecnología de la información y servicios a los estudiantes, que no eran vistos 
como ligados al proceso de enseñanza y de aprendizaje, y ahora son críticos para el suceso de la misión de esas instituciones. En ese sentido, Hitch y MacBrayne (2003) sugieren que las instituciones que ofrecen cursos en la modalidad de $\mathrm{EaD}$ deben considerar la implantación de sistemas informáticos para dar soporte tanto al proceso de enseñanza y aprendizaje, como a todos los servicios académicos. Esa propuesta, basada en el uso intensivo de TIC, sugiere ofrecer servicios personalizados, que deben ser diseñados con la perspectiva de los estudiantes, que son los usuarios finales de los referidos servicios.

Por lo tanto, el currículo como planificación, además de retomar y abarcar las concepciones de contenido, dado que inevitablemente en él debe estar incluido el contenido educativo, también señala el marco ideal dentro del cual se desarrollará la actividad educativa (Angulo, 1994).

\section{LA REALIDAD INTERACTIVA}

Retomando la discusión sobre los medios y el currículo, Ballesta Pagán (2011) defiende que lo importante es aunar, por un lado el estudio analítico de los medios, es decir, las características técnicas, posibilidades, funcionamiento, dominio de los lenguajes, con el examen curricular, o sea, su utilización, habilidades y destrezas, contenidos curriculares de comunicación audiovisual, procedimientos y formas de estructuración de la información. Eso puede ser hecho, según Gallego (1997) citado por Ballesta Pagán (2011), por dos vías. Por un lado, profundizando el conocimiento de las posibilidades intrínsecas de los medios de modo que se proporcionen pautas para su selección y utilización. Y por otro lado, examinando la necesidad que representan los medios para los profesores en su labor de diseminar conocimientos, actitudes o procedimientos concretos.

En la primera vía predominan los estudios e investigaciones sobre los medios, como acontece en los manuales clásicos sobre medios en la educación. La segunda vía es mucho más compleja y, sin duda, su desarrollo supone una mayor implicación educativa. La concepción de los medios como elementos curriculares implica que no pueden ser estudiados ni desconectados de los demás elementos. Averiguar cómo funcionan los medios en el contexto curricular pasa por conocer y profundizar el conocimiento de la práctica de los profesores con y sobre los medios. La praxis profesional, en tanto que conocimiento y acción, pasa por conocimientos, actitudes, valores y opiniones sobre los medios, así como las acciones y las formas de utilizarlos (Ballesta Pagán, 2011). 
Por lo tanto, si el currículo es determinado, y a la vez determina la vida de la institución de enseñanza, el conocimiento de cómo es vivido, creado y traducido de forma interactiva por docentes y alumnos en las clases resulta esencial para mejorar sus resultados (Clandinin y Cornelly, 1992). Stenhouse (1987) afirma que si el currículo es también, y fundamentalmente, lo que sucede en las salas de clase, es necesario una nueva perspectiva que centre su interés en las conexiones (o desconexiones), entre el currículo como intención y el currículo como acción a través del papel creativo del docente.

Los Ambientes Virtuales de Aprendizaje ofrecen diversos recursos para registrar y posteriormente analizar las consecuencias de la aplicación del currículo de cursos de Educación a Distancia. Un ejemplo consiste en la utilización por parte del profesor de un diario de abordo (Blog), para registrar los hechos, acontecimientos y experiencias, detectadas en el transcurso del proceso de enseñanza y aprendizaje. Por ejemplo, podrían ser registradas en el Blog cuestiones del tipo: ¿Qué actividades resultaron bien y cuáles no alcanzaron los objetivos trazados?, ¿Qué cuestiones en el debate de los foros tuvieron más aceptación por parte de los alumnos?, ¿Qué ejercicios de auto-evaluación fueron más utilizados y por qué?, ¿Cuáles son las principales dificultades de los alumnos a criterio de los tutores?

Estas y otras cuestiones pueden ser registradas periódicamente en el Blog para posterior análisis. A partir de ese análisis será posible reflexionar respecto de la aplicación práctica del currículo, y apuntar eventuales ajustes que se juzguen necesarios para equilibrar el currículo como intención y el currículo como acción.

\section{CONSIDERACIONES FINALES}

Uno de los problemas que enfrenta la educación en la actualidad es que los profesores son inmigrantes digitales, que hablan un anticuado lenguaje (de la era pre-digital) y se esfuerzan por enseñar a alumnos nativos digitales que hablan un lenguaje completamente nuevo. Si los educadores inmigrantes digitales realmente desean comunicarse con los nativos digitales, van a tener que cambiar (Prensky, 2001). Para eso, la incorporación innovadora de las TIC en los procesos de enseñanza y de aprendizaje es una estrategia que debe reforzarse. Esto supone configurar nuevos escenarios en las relaciones entre profesores, alumnos y en los contenidos que deben ser estudiados, y hacerlo también en la evaluación de todo el proceso de enseñanza y aprendizaje (Marchesi, 2009). 
Sin embargo, Bonilla (2003) alerta que las nuevas tecnologías no fueron concebidas para la educación, no aparecen naturalmente en los sistemas de enseñanza, no son demandadas por la comunidad docente, no se adaptan fácilmente al uso pedagógico y, muy probablemente en el futuro se desarrollarán de forma muy parcial en función de la demanda proveniente del sector educativo.

Eso planea uno de los problemas claves de la relación entre las políticas nacionales de educación y las TIC: la dificultad de implantar en la educación elementos que le son extraños, que no surgen ni se desarrollan dentro de los sistemas educativos, y que, por lo tanto, no se instalan en ellos de forma natural. Todos los contenidos curriculares son susceptibles de ser apoyados por el uso de tecnologías digitales, pero eso depende de la mediación pedagógica de los educadores, de su propio conocimiento y de la forma de gestionar el aprendizaje de los estudiantes a través de los recursos disponibles en su centro educativo y en su comunidad. La interacción de las TIC en el currículo es un desafío (Sunkel, 2009), así como lo es la adecuación del currículo para la Educación a Distancia soportada por TIC.

Por lo tanto, la capacitación de profesores para que dispongan de las competencias necesarias que les permitan incorporar de forma natural las TIC en su práctica pedagógica constituye la variable fundamental para garantizar el éxito del esfuerzo realizado. El análisis de las condiciones que facilitan la incorporación de las TIC en los procesos de enseñanza y de aprendizaje de forma innovadora se convierte en una reflexión ineludible (Marchesi, 2009).

\section{CONCLUSIONES}

Las nuevas generaciones de estudiantes, que nacieron a partir de la década de los años 90, crecieron y se desarrollaran utilizando TIC, especialmente las tecnologías digitales. De hecho, las TIC invadieron nuestro vivir cotidiano y, como no podría ser diferente, modificaron la forma en cómo las personas estudian y construyen su conocimiento. En particular, las TIC posibilitaron nuevos enfoques de la Educación a Distancia. Eso nos indica la necesidad de repensar las prácticas pedagógicas y de re-posicionar los medios en el contexto del currículo en $\mathrm{EaD}$.

A pesar de que la comprensión de lo que entendemos por currículo depende de conceptos variables, en este capítulo elegimos un marco de comprensión del currículo y con esa lente académica hemos intentado identificar los aspectos que debemos tener en cuenta para su elaboración en la EaD. Ese marco de comprensión considera el currículo como contenido o conocimiento a ser desarrollado, como la 
planificación que apunta el marco dentro del cual se desarrollarán las actividades educativas, y como la realidad interactiva que describe los significados, patrones de conducta, y valores morales que subyacen la experiencia educativa. Para cada una de esas tres dimensiones identificamos aspectos del currículo que, a nuestro entender, deben ser considerados en la Educación a Distancia.

En este trabajo, el debate sobre TIC y currículo se organizó no solo en torno de aprender sobre TIC, sino que, y principalmente, trató del aprendizaje con TIC, en particular, en Educación a Distancia. Sin embargo, no podemos olvidarnos que las TIC presentan determinados marcos de interpretación que pueden relativizar nuestro propio conocimiento (Ballesta Pagán, 2011). Por lo tanto, es necesario desmitificar las TIC y atribuirles el papel que les corresponde, pues a pesar que se considere importante el uso de la tecnología, conviene recordar que su utilización se torna desprovista de sentido si no está aliada con una perspectiva educativa comprometida con el desarrollo humano, con la formación de ciudadanos, con la gestión democrática, con el respeto a la profesión del profesor y con la calidad social de la educación (André, 2009).

\section{REFERENCIAS BIBLIOGRÁFICAS}

Alonso, C. M.; Gallego D. J.; Honey, P. (2007). Estilos de Aprendizaje. Bilbao: Mensajero.

Anaraki, F. (2004). Developing an effective and efficient e-learning platform. International Journal of the Computer, the Internet and Management, 12 (2), (57-63).

André, C. F. (Org.) (2009). Guia de tecnologias educacionais. Brasília: Ministério da Educação, Secretaria de Educação Básica.

Angulo, J. F. (1994). ¿A qué llamamos currículum? En: Ángulo, J. F.; Blanco, N. Teoría y desarrollo del currículum. Archidona (Málaga): Ediciones Aljibe.

Ballesta Pagán, J. (2011). Los medios en la enseñanza. En: Sevillano García, M. L. Medios, recursos didácticos y tecnología educativa. Madrid: Pearson.

Bloom, B. S. (1956). Taxonomy of educational objectives: the classification of educational goals.
Handbook I: Cognitive Domain. New York: McKay.

Bonilla, J. (2003). Políticas nacionales de educación y nuevas tecnologías: el caso de Uruguay. Educación y Nuevas Tecnologías. Experiencias en América Latina. Buenos Aires: IIPE - UNESCO.

Boud, D.; Keogh, R.; Walker, D. (1985). Reflection, turning experience into learning. London; New York: Kogan Page; Nichols Pub.

Breitner, M. H.; Hoppe, G. (2005). A glimpse at business models and evaluation approaches for electronic(e)learning. E-learning Einsatzkonzepte und Geschäftsmodelle, 1, (179-193).

Candy, Ph. (1991). Self-direction for lifelong learning: a comprehensive guide to theory and practice. San Francisco, California: Jossey-Bass Higher and Adult Education Series. 
Clandinin, D. J.; Connelly, F. M. (1992). Handbook of research on curriculum. New York: Mc Millan.

Costa Sousa, J.; Bruno-Faria, M. F.; de Almeida e Carmo, H. D. (2012). Proceso de innovación en la gestión de sistemas de educación a distancia: relevancia y estado del arte. RIED. Revista Iberoamericana de Educación a Distancia, 15 (2), (95-122).

Downes, St. (2004). Ten years after. [en línea] Disponible en: http://www. downes.ca/files/TenYearsAfter.ppt (consulta 2006, 7 de julio).

Fischer, G.; Scharff, E. (1998). Learning technologies in support of self-directed learning. Journal of Interactive Media in Education, 98 (4).

Fox, M. (2005). Kineo Insight: 50 ideas for free e-learning. [en línea] Disponible en: www.kineo.co.uk (consulta 2006, 7 de julio).

García Cué, J. L. (2006). Los estilos de aprendizaje y las tecnologías de la información y la comunicación en la formación del profesorado. Madrid: UNED Tesis doctoral.

Garrison, D. R.; Anderson, T. (2003). E-learning in the 21st century: $a$ framework for research and practice. London: RoutledgeFalmer.

Gimeno Sacristán, J. (2002). La educación que tenemos, la educación que queremos. In: La Educación en el siglo XXI: los retos del futuro inmediato. $4 \mathrm{a}$ Ed. Barcelona: Editorial Graó.

Gimeno Sacristán, J.: Pérez Gómez, A. I. (1998). Compreender e transformar o ensino. São Paulo: Artmed.

Hitch, L. P; MacBrayne, P. (2003). A model for effectively supporting e-learning. The Technology Source Archives at the University of North Carolina. [en línea] Disponible en: http:// technologysource.org/article/model for effectively supporting elearning (consulta 2007, 5 de junio).
Ho, C.; Swan, K. (2007). Evaluating online conversation in an asynchronous learning environment: an application of Grice's cooperative principle. Internet and Higher Education, 10, (3-14).

Honey, P.; Mumford, A.(2000). The learning styles helper's guide. Maidenhead-UK: Peter Honey Publications.

Iriondo Otero, W. R. (2008). Educação a distância: desenvolvimento de habilidades cognitivas de alto nível em e-learning. Tese de doutorado. Florianópolis: PPEGC/UFSC.

Iriondo Otero, W. R.; Ribeiro, L. M. (2010). Avaliação de interações assíncronas baseadas em texto em Ambientes Virtuais de Ensino Aprendizagem. VII ESUD - Congresso Brasileiro de Ensino Superior a Distância. Cuiabá: Universidade Federal de Mato Grosso - UFMT.

Jacinski, E.; Faraco, C. A. (2002). Tecnologias na Educação: uma solução ou problema pedagógico. Revista Brasileira de Informática na Educação, Porto Alegre - RS, 10 (2), (49-56).

JISC - The Joint Information Systems Commitee (UK). (2004). Effective practice with e-learning: a good practice guide in designing for learning. Bristol: JISC development group. [en línea] Disponible en: www.jisc.ac.uk/ elearning (consulta 2007, 1 de octubre).

Laurillard, D. (2002). Rethinking university teaching: a conversational framework for the effective use of learning technologies. 2 Ed. London: Routledge Falmer.

Long, H. B. (1990). Changing concepts of self-direction in learning. En: H. B. Long \& Associates Advances in research and practice in self-directed learning, 1-8. Norman, OK: Oklahoma Research Center for Continuing Professional and Higher Education.

Marchesi, Á. (2009). Preámbulo. En: Carneiro, R.; Toscano, J. C.; Díaz T. 
Metas Educativas 2021: Los desafíos de las TIC para el cambio educativo. Madrid: Fundación Santillana.

Martínez Geijo, P. (2007). Aprender y enseñar. Los estilos de enseñanza desde la práctica de aula. Bilbao: Mensajero.

Melaré, D. (2011). Estilos da Aprendizagem na Atualidade. [en línea] Disponible en: http://estilosdeaprendizagemvolo1.blogspot.com (consulta 2011, 23 de octubre).

Moreira, A.; Candau, V. (2007). Indagações sobre currículo: currículo, conhecimento e cultura. Brasília: Ministério da Educação, Secretaria de Educação Básica.

Padilha, M. (2009). Tipos de indicadores: una mirada reflexiva. En: Carneiro, R.; Toscano, J. C.; Díaz T. Metas Educativas 2021: Los desafíos de las TIC para el cambio educativo. Madrid: Fundación Santillana.

Pask, G. (1976). Conversation theory: applications in education and epistemology. Amsterdam: Elsevier.

Prensky, M. (2001). On the Horizon. $M C B$ University Press, 9 (5).
Robinson, A.; Udall, M. (2003). Developing the Independent Learner: The Mexican Hat Approach. 3rd International Symposium on Engineering Education. Southampton, UK.

Rosa, M.; Maltempi, M. V. (2006). A avaliação vista sob o aspecto da educação a distância. Ensaio: aval.pol. públ.Educ, 14 (50), (57-76). [en línea] Disponible en: http://www.scielo.br/ cgi-bin/wxis.exe?cal/?IsisScript=iah (consulta 2007, 23 de octubre).

Simpson, O. (2002). Supporting students in online, open and distance learning. 2nd Ed. London: Kogan Page.

Stenhouse, L. (1987). Investigación y desarrollo del currículum. $2 \mathrm{a}$ Ed. Madrid: Morata.

Sunkel, G. (2009). Las TIC en la educación en América Latina: visión panorámica. En: Carneiro, R.; Toscano, J. C.; Díaz T. Metas Educativas 2021: Los desafíos de las TIC para el cambio educativo. Madrid: Fundación Santillana.

\section{PERFIL ACADÉMICO Y PROFESIONAL DE LOS AUTORES}

Walter Ruben Iriondo Otero. Profesor del Centro de Educación a Distancia, en la Universidad Federal de Pelotas (Brasil). En su tesis de doctorado, realizada en Brasil y en el Reino Unido, investigó el desarrollo de habilidades cognitivas en Educación a Distancia. En 2009 realizó postdoctorado en la Universidad Técnica de Lisboa (Portugal) y en 2012 investigó aspectos del uso de las TIC en la educación, en la Universidad Nacional de Educación a Distancia (España).

E-mail: walter.iriondo@upel.edu.br

Domingo J. Gallego Gil. Profesor de la Universidad Nacional de Educación a Distancia (España). Doctor en Filosofía y Letras por la Universidad Complutense de Madrid y Master en Educational Tecnology and Communication, Columbia University, New York. Su tesis se centró en la Psicosociología del Medio Telefónico en la Enseñanza a Distancia. Ha dirigido 71 tesis doctorales. Ha dirigido proyectos 
de enseñanza a distancia en Argentina, Chile, Ecuador, Paraguay, Brasil, Perú, Colombia, Costa Rica.

E-mail: domingoj.gallego@gmail.com

DIRECCIÓN DE LOS AUTORES

Prof. Dr. Walter R. Iriondo Otero

Universidad Federal de Pelotas

Centro de Educación a Distancia

Rua Gomes Carneiro, 1 - Centro

Pelotas - RS - 96010.610 - Brasil

Prof. Dr. Domingo José Gallego Gil

Universidad Nacional de Educación a Distancia

Facultad de Educación

Despacho 225

Juan del Rosal, 14

Madrid - 28040 - España

Fecha de recepción del artículo: 07/02/2012

Fecha de aceptación del artículo: 18/09/2012

\section{Como citar este artículo:}

Iriondo Otero, W.; Gallego Gil, D. (2013). El currículo y la educación a distancia. RIED. Revista Iberoamericana de Educación a Distancia, volumen 16, $\mathrm{n}^{0}$ 1, pp. 109-132. 